\title{
Parametric studies and optimisation of pumped thermal electricity storage
}

\author{
Joshua McTigue $^{\mathrm{a}}$, Alexander White ${ }^{\mathrm{a},{ }^{,} \text {, and Christos N. Markides }}{ }^{\mathrm{b}}$ \\ ${ }^{\text {a }}$ Cambridge University Engineering Department, Trumpington Street, Cambridge CB2 1PZ, United Kingdom \\ ${ }^{\mathrm{b}}$ Department of Chemical Engineering, Imperial College London, London SW7 2AZ, United Kingdom \\ * Corresponding author. Email: ajw36@cam.ac.uk; Tel. +44 (0)1223 765310; Fax.+ 44 (0)1223 765311
}

\begin{abstract}
Several of the emerging technologies for electricity storage are based on some form of thermal energy storage (TES). Examples include liquid air energy storage, pumped heat energy storage and, at least in part, advanced adiabatic compressed air energy storage. Compared to other large-scale storage methods, TES benefits from relatively high energy densities, which should translate into a low cost per MWh of storage capacity and a small installation footprint. TES is also free from the geographic constraints that apply to hydro storage schemes. TES concepts for electricity storage rely on either a heat pump or refrigeration cycle during the charging phase to create a hot or a cold storage space (the thermal stores), or in some cases both. During discharge, the thermal stores are depleted by reversing the cycle such that it acts as a heat engine. The present paper is concerned with a form of TES that has both hot and cold packed-bed thermal stores, and for which the heat pump and heat engine are based on a reciprocating Joule cycle, with argon as the working fluid. A thermodynamic analysis is presented based on traditional cycle calculations coupled with a Schumann-style model of the packed beds. Particular attention is paid to the various loss-generating mechanisms and their effect on roundtrip efficiency and storage density. A parametric study is first presented that examines the sensitivity of results to assumed values of the various loss factors and demonstrates the rather complex influence of the numerous design variables. Results of an optimisation study are then given in the form of trade-off surfaces for roundtrip efficiency, energy density and power density. It is concluded that roundtrip efficiencies approaching those for pumped hydro schemes might be achievable whilst simultaneously attaining energy storage densities of around $200 \mathrm{MJ} \mathrm{m}^{-3}$, but this is contingent upon attaining compression and expansion efficiencies for reciprocating devices that have yet to be proven.
\end{abstract}

Keywords: electricity storage; thermal energy storage; irreversibility; heat transfer; optimisation. 


\section{Introduction}

The finite nature of fossil fuel reserves together with a wide range of health and environmental concerns arising from the release of combustion products have been acting as drivers for the increasing uptake of renewable sources of energy, such as solar and wind [1]. These energy sources have the potential to reduce the overall dependence on fossil fuels and the emissions arising from their use, however, both solar (especially PV) and wind energy are associated with variable, intermittent and (particularly for wind) uncertain outputs. Beyond the economic considerations of using a significant fraction of inherently variable power generation, the intermittent nature of these energy sources has given rise to concerns regarding their reliable integration into the electric grid. These factors have led to the widely accepted recognition that energy storage forms an essential part of efficient and sustainable future energy systems, in particular ones featuring significant amounts of renewable resources. In the UK, for example, it is estimated that over the next few decades the integration of intermittent sources into the power infrastructure will require storage capacities of the order of hundreds of GWh - an order of magnitude greater than current capacity [2].

Pumped hydro storage (PHS) is currently the dominant large-scale energy storage technology, with over $99 \%$ of the world's installed storage capacity in this form. However, the high initial cost and geographical constraints of PHS mean that many new technologies are emerging, including batteries, flow batteries, compressed air storage (CAES) and, of particular interest here, thermal energy storage (TES). A comprehensive review of these technologies is given in Ref. [3]. TES systems suitable for large-scale storage (i.e., $>100 \mathrm{MWh}$ ) include: cryogenic systems for which energy is stored within tanks of liquid air or liquid nitrogen; pumped heat storage where energy is stored in high temperature reservoirs, either as 'sensible heat' or 'latent heat'; and hybrid systems that simultaneously exploit both hot and cold thermal storage. Proposals for new types of CAES (notably 'Advanced-Adiabatic' CAES, or AA-CAES) also include a thermal storage component. Despite the variety of detailed arrangements, all TES systems effectively make use of some form of heat pump during the charge phase to extract thermal energy from a low temperature source and deliver it (together with the energy from the electrical work input) to a higher temperature sink. Energy flows are then reversed during discharge such that the system operates as a heat engine. Since the maximum (Carnot) efficiency of the heat engine is precisely the reciprocal of the maximum coefficient of performance of the heat pump, the round-trip efficiency is limited only by the reversibility of the system's various processes.

The present paper focuses on a form of TES system referred to here as 'pumped thermal' electricity storage (PTES) ${ }^{*}$, several independent patents for which seem to have emerged almost simultaneously [4]-[7]. A similar system also seems to have been proposed much earlier [8]. For the particular variant of PTES considered here, based mainly on that described in Ref. [6], the charging (heat pumping) phase is achieved by an electrically driven reverse Joule-Brayton cycle, which establishes a temperature difference between two packed-bed thermal stores. Electrical energy is thus converted to thermal energy that then resides in the stores. When electricity is required, the cycle operates in forward (heat engine) mode, returning heat from the hot to the cold store, thereby recovering electrical work.

The important factors in determining the merit of any electrical energy storage technology are its round-trip efficiency (i.e., the fraction of electrical energy input retrieved upon discharge) and its capital costs per MW installed capacity and per MWh of storage. In this respect, PTES benefits from relatively high energy density, which implies a small plant footprint and low capital cost per MWh. (Comparison of a few large-scale storage

\footnotetext{
*Also known as 'pumped heat' electricity storage (PHES)
} 
technologies suggests that PTES might achieve an energy density roughly an order of magnitude greater than that for CAES and two orders of magnitude greater than for PHS [9].) However, power density (as opposed to energy density) is also an important factor as it impinges on the size and cost of the machinery and hence on the cost per MW. Table 1 compares power densities (in terms of exergetic flow rate per unit volumetric flow rate of working fluid) for a few different technologies. The figure for PTES is relatively low, suggesting that controlling pressure losses and designing small, cheap power conversion machinery for such a system will be challenging.

Previous theoretical work relating to PTES includes 'endoreversible' analysis of a generic system [10] and more practical studies of open [8] and closed-cycle devices [11] based on turbomachinery. For the system proposed in Ref. [6] however, compression and expansion are achieved by reciprocating devices since there is evidence that these are capable of higher polytropic efficiencies than turbomachinery. A second, crucial advantage of reciprocating devices is that, by adjusting valve timings, they can in principle be reversed such that compressors become expanders and vice versa. This means that the same devices can be used for both charge and discharge, thereby lowering the capital cost per MW and enabling a rapid switch to meet sudden increases in electricity demand.

A 1.5 MW and $6 \mathrm{MWh}$ system based on reciprocating devices is currently being developed in the UK funded by the Energy Technologies Institute (ETI) ${ }^{*}$ and its proponents estimate that it will attain an overall round-trip efficiency of $75 \%$. Achieving this will require extremely low 'thermodynamic' losses in the compressors and expanders, and in the other system components, notably the thermal stores. The objectives of the present paper are to estimate the feasibility of such low losses, to examine the sensitivity of system performance to the various loss parameters and to determine also the role of different operating and geometric parameters. In the following sections an account is first presented of the loss-generating mechanisms that occur throughout a PTES system. Sub-models for these components are then described and brought together into overall system calculations, which are presented in the form of parametric studies. These serve to emphasise the interplay between the different sources of loss, and how storage density and the distribution of exergetic losses depend on the various system pressures and temperatures and on other, geometric factors. Although such parametric studies provide a sound physical basis for guiding design, system optimisation remains a complex task due to the large number of operational and design parameters, the multiple and often conflicting objectives (e.g. efficiency and energy/power density) and the uncertainty associated with some aspects of the loss modelling. An evolutionary-based optimisation algorithm has thus been applied to identify promising designs. This algorithm allows trade-off surfaces (also known as a Pareto fronts) to be plotted, thereby giving insight into how the optimal design may vary when multiple objectives are considered.

\section{Baseline Design}

The outline design features of a hypothetical 2 MW PTES system with 16 MWh of storage is given in Ref. [12]. A system of this size has been adopted for the analysis presented here and it is useful to first provide estimates for the main system parameters. The power and storage capacity given below are 'nominal' values in the sense that they are the values that would be achieved in the absence of losses and in the (hypothetical) case where the reservoirs can be fully charged.

\footnotetext{
*See http://www.eti.co.uk//technology_programmes/energy_storage_and_distribution
} 


\subsection{Operating pressures and temperatures}

Figure 1 shows the basic layout of a PTES system, together with T-s (temperature-entropy) diagrams for typical discharge and charge processes, which follow the standard and reverse Joule cycle respectively. The main system components are two compression-expansion devices (CE and EC) and two thermal reservoirs (one hot, HR, and one cold, CR). Following [6], the discharged state of the reservoirs is set close to ambient temperature: $310 \mathrm{~K}$ in the present case. With argon as the working fluid (as proposed in Ref. [5]), and with a pressure ratio of 10:1, the nominal hot and cold storage temperatures (based on isentropic compression and expansion) are then $778 \mathrm{~K}$ and $123 \mathrm{~K}$ respectively.

\subsection{Reservoir sizing}

For a reversible, adiabatic PTES system, the stored energy that can be converted back to useful work (i.e., the 'available' energy) is simply the difference between the stored internal energies of the two reservoirs,

$$
E=M_{s}^{h} c_{s}^{h}\left(T_{2}-T_{3}\right)-M_{s}^{c} c_{s}^{c}\left(T_{1}-T_{4}\right)=M_{s}^{h} c_{s}^{h}\left(T_{2}-T_{3}-T_{1}+T_{4}\right),
$$

where $M_{s}$ is the mass of storage material, $c_{s}$ is its average specific heat capacity over the relevant temperature range, and the superscripts $h$ and $c$ refer to the hot and cold reservoirs respectively. The right hand equality in Eq. (1) arises from the requirement that the two reservoirs should charge in the same period and must therefore have the same heat capacity. As discussed in Ref. [13], $\mathrm{Fe}_{3} \mathrm{O}_{4}$ provides a suitable storage material due to its high heat capacity per unit volume and its low fractional variation of heat capacity over the temperature ranges of interest. Data for $\mathrm{Fe}_{3} \mathrm{O}_{4}$ are given in Table 2, together with the required storage masses computed from Eq. (1) for $16 \mathrm{MWh}$ of storage. The reservoir volumes are also given in the table, calculated on the basis that the storage material is in the form of a packed bed of spherical pebbles with an average void fraction of 0.35 . Note that these volumes would correspond to 'square' (i.e., $L / D=1$ ) cylinders with internal diameters of $4.5 \mathrm{~m}$ and $5.3 \mathrm{~m}$ for the hot and cold stores respectively.

\subsection{Compression and expansion device sizing}

The power output for a reversible, adiabatic PTES system is given by,

$$
\dot{W}_{\mathrm{x}}=\dot{m} c_{p}\left\{\left(T_{2}-T_{1}\right)-\left(T_{3}-T_{4}\right)\right\} .
$$

Using the temperatures listed above and data for $\operatorname{argon}\left(c_{p}=520 \mathrm{~J} \mathrm{~kg}^{-1} \mathrm{~K}^{-1}\right)$, the mass flow rate required for a $2 \mathrm{MW}$ device comes to $13.7 \mathrm{~kg} \mathrm{~s}^{-1}$. The total swept volumes for the compression-expansion cylinders are computed from,

$$
\dot{m}=\rho_{\text {ref }} V_{s} \eta_{v} \omega / 2 \pi,
$$

where the reference density $\rho_{\text {ref }}$ is at inlet for compression but at exit for expansion - i.e., $\rho_{1}$ for $\mathrm{CE}$ and $\rho_{4}$ for EC. The volumetric efficiency $\eta_{v}$ is a function of the pressure ratio, $\beta$, and clearance volume ratio of the cylinders $\left(V_{\min } / V_{\max }\right)$. With the latter set at 0.05 , the simplified model presented in Appendix A for an ideal reciprocating device gives a volumetric efficiency of 0.84 at $\beta=10$. The resulting swept volumes are shown in Table 3, together with various cylinder dimensions computed on the basis of 6-cylinder devices running at 1200 RPM, with each cylinder having an aspect ratio (stroke/diameter) of 0.25 . This low aspect ratio is proposed in the designs described in Ref. [12] on the grounds that the resulting low piston velocity will give low valve pressure losses and low inertial loading. 


\subsection{Other design considerations}

In addition to the main components described above, the PTES system also requires heat exchangers (HX1 and HX3 in Fig. 1), and a buffer vessel (BV in the figure). The heat exchangers are needed to counter the effects of irreversibility throughout the system and their size can only be determined, therefore, after consideration of the cycle efficiency. The buffer vessel is required because the total mass of gas within the two reservoirs changes during charge. The total change between fully charged and fully discharged for the nominal design is $142 \mathrm{~kg}$, as indicated from the figures in Table 2 . This requires a buffer volume of $8.7 \mathrm{~m}^{3}$ if situated at Point 3 in the cycle, as in Fig. 1.

\section{System and Component Modelling}

In order to determine the influence of the various system parameters on round trip efficiency, power density and storage density, a simple system model has been developed based on quasi-steady analysis of each of the system components. Heat exchangers, compressors and expanders are treated as steady flow devices (in the time-averaged sense), but the equations governing heat transfer within the reservoirs are integrated in time in order to track the hot and cold thermal fronts. This is necessary because the stored available energy and the exergetic losses in the reservoirs are dependent upon the time-history of their operation, as described in Refs. [13,14]. For the other components, estimates are first made for various loss parameters, based on the nominal design described above. Several of these parameters are, however, subject to considerable uncertainty, either because they depend on detailed design (e.g., pressure losses within pipework) or because the underlying theory has not yet been sufficiently developed (e.g., for compression and expansion efficiencies). The approach adopted is therefore to undertake baseline calculations using best estimates for minimum values of these parameters and then to examine the impact of their variation on the overall performance.

\subsection{Compression and expansion losses}

The simplest approach for modelling 'steady flow' compressors and expanders is by either an isentropic or polytropic efficiency. For turbomachines, published data suggest polytropic efficiencies (i.e., infinitesimal stage efficiencies) of about $90 \%$ are achievable. For reciprocating devices, data is scant but compression efficiencies in the range $75-85 \%$ seem typical [15]. Although this is lower than the values cited for turbomachinery, much of the loss in reciprocating devices is associated with valve pressure drop and there may be scope for considerable improvement. On the other hand, the relatively long residence time of the gas within the device (compared to a turbo-compressor) means that there is often significant heat transfer to the surroundings and hence isentropic efficiency is ill-defined. As shown in Ref. [16], the effects of heat leakage and irreversibility can, however, be distinguished by combining a heat leakage factor (defined as the ratio between the instantaneous heat and work transfer, $\alpha=\mathrm{d} q / \mathrm{d} w_{x}$ ) with a polytropic efficiency (defined as $\eta=-v \mathrm{~d} p / \mathrm{d} w_{x}$ ). This leads to polytropic relations of the form,

$$
\tau_{\mathrm{c}}=\beta_{\mathrm{c}}^{\phi_{\mathrm{c}}} \quad \text { with } \quad \phi_{\mathrm{c}}=\frac{\gamma-1}{\gamma}\left(\frac{1-\alpha_{\mathrm{c}}}{\eta_{\mathrm{c}}}\right),
$$

where $\tau_{\mathrm{c}}$ and $\beta_{\mathrm{c}}$ are the compressor temperature and pressure ratios respectively. Compressor work input is then found by straightforward application of the steady flow energy equation, giving,

$$
w_{c}=\frac{c_{p} T_{1}\left(\tau_{\mathrm{c}}-1\right)}{1-\alpha_{\mathrm{c}}}
$$


where $T_{1}$ here is the compressor inlet temperature. Similar expressions to (4) and (5) apply to expansion processes. Although this approach allows the impact of irreversibility and heat leakage to be studied separately, the fundamental difficulty lies in estimating values for $\alpha$ and $\eta$. In theory, with sufficient insulation, $\alpha$ could be reduced to zero, but $\eta$ is difficult to estimate. The chief losses are likely to be: (i) pressure drop through the valves; (ii) cyclic heat transfer to and from the cylinder walls across finite temperature differences and (iii) mixing of fresh intake gas with the residual gas within the clearance volume. It is convenient to treat pressure losses separately as throttling losses external to the compression process, and so $\eta$ is used here solely to represent heat transfer and mixing irreversibility. Rudimentary estimates of the minimum values of loss factors that might be achieved are given in Appendix A.

\subsection{Pressure losses}

Pressure losses in valves, pipework, heat exchangers and the reservoirs all contribute to the expander seeing a lower pressure ratio than the compressor. These losses are represented here by fractional pressure loss factors, $f_{p}=\Delta p / p$, since these are most closely tied to exergetic losses. Thus a given absolute pressure loss $\Delta p$ has a greater impact on performance if it occurs in the low-pressure part of the cycle. Estimates of pressure losses through the compressor and expander valves are given in Appendix A, but losses within manifolds, ducts and heat exchangers etc. cannot easily be estimated without knowledge of the detailed geometry, and so a range of $f_{p}$ values has been considered. It is worth noting, however, that if pressure losses are assumed to be of the order of $1 / 2 \rho v^{2}$, and if these are to be kept within $1 \%$ of the local pressure then this would imply ducts of the order of $0.5 \mathrm{~m}$ diameter at Point 1 (Fig. 1) for the nominal design. Pressure losses in the reservoirs are treated separately and calculated explicitly, as discussed below.

\subsection{Thermal reservoir losses}

The main sources of loss within the reservoirs are frictional pressure loss and heat transfer (thermal) irreversibility. Heat leakage is not considered here since, with sufficient insulation, it can be reduced to any desired level. However, estimates suggest that a $1 \%$ heat leakage to or from each reservoir would typically reduce round-trip efficiency by about $2 \%$ since leakage reduces both the stored energy and the Carnot efficiency at which that energy can be converted to work [9].

Thermal losses occur because heat exchange necessarily takes place across a finite gas-solid temperature difference. The model used to quantify these losses is based on the well-established Schumann model of heat transfer in packed beds [17], which assumes that the flow is one-dimensional and that heat transfer is limited by surface effects (i.e., the internal thermal resistance of particles is negligible). The result is that the energy equations for the gas and solid can be expressed approximately as,

and

$$
\frac{\partial T_{g}}{\partial z}=\frac{T_{s}-T_{g}}{\ell}
$$

where the length and time scales are defined by,

and

$$
\begin{gathered}
\ell=\frac{1}{\operatorname{St}(1-\varepsilon) S_{v}}, \\
\tau=\frac{\rho_{s} c_{s}}{\operatorname{St} c_{p} G S_{v}} .
\end{gathered}
$$


In these expressions, $G$ is the mass flow rate of gas per unit (open-tube) area, $\mathrm{St}$ is the Stanton number (based on the open-tube velocity) and $S_{v}$ is the particle surface-to-volume ratio, which is $6 / d_{p}$ for spherical particles. In practice, a number of refinements to this model are included to account for unsteady accumulation terms for the gas, temperature dependence of the solid heat capacity, and variations in density, viscosity and other gas properties. Most of these have only a minor impact, but the variation of $c_{s}$ can significantly affect the thermal losses. Full details of the numerical method are given in Refs. [13] and [14], and typical temperature profiles for a cold reservoir are shown in Fig. 2. The instantaneous entropy generation rate (and hence the thermal loss) is determined from the temperature profiles as,

$$
\dot{S}_{i r r}=\int\left(\frac{1}{T_{s}}-\frac{1}{T_{g}}\right) d \dot{Q}=(1-\varepsilon) A S_{v} \int_{0}^{L} h \frac{\left(T_{g}-T_{s}\right)^{2}}{T_{s} T_{g}} d z,
$$

where $h$ is the local heat transfer coefficient and $A$ is the (open-tube) area of the packed bed. Previous work has established that the fractional exergetic loss decreases with the dimensionless length of the reservoir $(\Lambda=L / \ell)$ and depends in a rather complicated manner on the inlet and exit temperatures. For periodic, cyclic operation (as opposed to one-off charge and discharge), thermal losses are also a function of the charge period expressed in the dimensionless form $\Pi=t_{\text {chg }} / t_{\text {nom }}$, where $t_{\text {nom }}=\Lambda \tau$ is the nominal charge time. This parameter is referred to here as the 'utilisation factor' since it is approximately equal to the stored energy as a fraction of its full-charge value.

Pressure losses in packed beds are relatively straightforward and computed from,

$$
\frac{d p}{d z}=\frac{S_{v}(1-\varepsilon) C_{f} G^{2}}{2 \varepsilon^{3} \rho_{g}}
$$

where $C_{f}$ is the friction coefficient obtained from packed-bed correlations such as those given in Ref. [18].

\subsection{Heat exchanger and other losses}

Geometric and other design details of the heat exchangers have not been included at this stage. Instead datum temperatures at Points 1 and 3 in the cycle are specified for each calculation and the heat exchange required to maintain these temperatures is then computed. As noted above, heat rejection must occur in order to counter irreversibility throughout the cycle and, because it occurs at above the environment temperature, there is a further exergetic loss associated with the heat exchangers themselves. In addition, there is a small throttling loss associated with returning the buffer volume gas to the low-pressure part of the cycle. Estimates indicate that this is very small and it has been neglected in these calculations.

\section{Parametric Studies}

Before presenting an optimisation study, it is useful to examine how the various system parameters impinge upon overall performance. Loss factors, operating conditions and geometric parameters have all been varied over the ranges shown in Table 4. As each quantity is varied, all others listed in the table are held at their nominal values, except when varying pressure ratio, as discussed further below. The effect on performance is considered in terms of:

Round trip efficiency:

$$
\chi=\frac{\text { net work output }}{\text { net work input }}=\frac{W_{\mathrm{dis}}^{\text {net }}}{W_{\text {chg }}^{\text {net }}}
$$

Energy density:

$$
\rho_{\mathrm{E}}=\frac{\text { net work output }}{\text { reservoir volume }}=\frac{W_{\mathrm{dis}}^{\text {net }}}{V_{\mathrm{CR}}+V_{\mathrm{HR}}}
$$


Power density:

$$
\rho_{\mathrm{P}}=\frac{\text { average power output }}{\text { hot cylinder volume }}=\frac{W_{\mathrm{dis}}^{\mathrm{net}} / t_{\mathrm{dis}}}{V_{s}^{\mathrm{CE}}}
$$

The net work terms in the above are obtained by integrating the power input or output over the duration of the charge-discharge cycle. (Some variation of power occurs because the hot and cold fronts reach the exits of the reservoirs.) Note that the round-trip efficiency considered here reflects only the thermodynamic component of loss since electrical and mechanical losses will also occur. For a $2 \mathrm{MW}$ machine using an induction motor-generator, electrical efficiencies of 97\% (in each direction) are commonplace, but mechanical losses for a custom-built reciprocating compressor-expander are less easy to estimate. Howes [12] suggests that a mechanical efficiency of $92 \%$ (again in each direction) might be possible, which together with electrical losses would give an overall round-trip efficiency of $\left(\eta_{\text {mech }} \eta_{\text {elec }}\right)^{2} \chi \approx 0.8 \chi$.

Due to space constraints, power density is not discussed in this section, but it is taken into account during the optimisation process presented in Section 5.

\subsection{Sensitivity to the assumed values of loss parameters}

Figure 2 shows the thermodynamic round-trip efficiency, $\chi$, as a function of each of the loss factors. The trends are in line with the simplified analysis presented in Ref. [9], except that a slightly greater sensitivity to heat leakage is predicted here. (Detailed scrutiny of the loss distribution suggests that this is associated with the 'exit loss', which was not accounted for in Ref. [9] and which occurs when the hot and cold fronts reach the reservoir exit, as indicated in Fig. 2.) The sensitivity to $\alpha$ and $\eta$ are both very significant, showing the importance of minimising heat leakage and emphasising the need for a better understanding of thermal irreversibility in reciprocating devices. In what follows it is assumed that $\eta$ is independent of operating conditions, but arguably it should fall with pressure ratio, as described in Appendix A.

\subsection{Variation of performance with operating conditions}

Figure 4 shows the effect of various cycle operating conditions on efficiency and power density. In varying these parameters, the nominal power and storage capacity have been held constant by adjusting the size of reservoirs and the mass flow rate. Pressure losses in the reservoirs are then adjusted in accord with Eq. (11), but the nominal $f_{p}$ values have been retained for valve, pipework and manifold losses.

At fixed pressure ratio, the efficiency and energy density are both improved by either increasing $T_{1}$ or decreasing $T_{3}$. Increasing the pressure ratio at fixed $T_{1}$ and $T_{3}$ has a similar effect, as shown in Fig. 4(a). These trends are consistent with the findings reported in Ref. [9] where it was argued that, if compression and expansion losses dominate, then the efficiency is governed mainly by the ratio $T_{2} / T_{3}$ since this determines the ratio between compression and expansion work. Increasing this ratio (as all of the above variations in $T_{1}, T_{3}$ and $\beta$ will achieve) thus makes the cycle less susceptible to compression and expansion losses. However, improvements along these lines will almost certainly result in increased costs and technical challenges since they imply higher top temperatures or pressures, or lower bottom temperatures. A more detailed examination of the distribution of losses also reveals that the benefits of higher work ratio are partly offset by increased thermal losses in the reservoirs. If the maximum and minimum cycle temperatures $\left(T_{2}\right.$ and $\left.T_{4}\right)$ are fixed, instead of fixing $T_{1}$ and $T_{3}$, then variation of the pressure ratio gives trends similar to those observed for gas turbine cycles for which there are optimum pressure ratios, as shown in Fig. 4(b). The optimum pressure ratios for energy density and efficiency are, in this case, 7.6 and 9.1 respectively, but the corresponding values of $T_{3}\left(3.5\right.$ and $\left.24.5^{\circ} \mathrm{C}\right)$ lie below ambient temperature. This precludes the possibility of rejecting heat in $\mathrm{HX} 3$, making it difficult to manage the thermal fronts as they emerge from the reservoirs. 
Figure 4(b) also shows the influence of 'utilisation factor' $\Pi$ and the discharge pressure ratio. Increasing $\Pi$

\subsection{Variation of performance with geometric factors}

The geometric parameters varied here are the hot and cold reservoir aspect ratios $(L / D)$ and particle heat exchange processes, between heat transfer losses and pressure losses. For example, small particles give large heat exchange area but increase the frictional effects. Likewise, long reservoirs give lower thermal losses [14], but the associated reduction in cross-sectional area implies higher fluid velocities and hence higher pressure drop. Due to the lower average gas density, pressure losses are more significant in the cold reservoir and hence the optimum aspect ratio is lower and optimum particle size larger than for the hot reservoir. However, it should be recalled that these results have been obtained with all other parameters fixed at their nominal values, and the picture changes when overall system optimisation is considered.

\section{Preliminary Optimisation Studies}

The design space for a PTES system is multi-dimensional and is possibly multi-modal and disjoint due to the large number of design variables, objectives and constraints. This makes optimisation by systematic parameter variation a complex task. A stochastic optimisation algorithm has therefore been applied to identify promising designs. The routine chosen for this purpose is a 'non-dominated sorting genetic algorithm' (NSGA-II) as described in Ref. [19]. Like other stochastic methods, this is well suited to the current problem as it is able to traverse the entire design space with limited risk of becoming trapped in local optima.

Full optimisation clearly requires consideration of economic factors, material limits, and other practical issues that lie outside the scope of the present paper. Nonetheless, a preliminary indication of useful design trends can be obtained on the basis of thermodynamics alone by simultaneously maximising the round trip efficiency and the energy and power densities. (Strictly, the optimiser minimises the negative of each of these quantities.) Since increasing cost is associated with higher pressures and extremes of temperature, limits are set for $\beta, T_{2}$ and $T_{4}$. Likewise, a lower limit is set on both $T_{1}$ and $T_{3}$ of $10{ }^{\circ} \mathrm{C}$ above the ambient temperature in order that $\mathrm{HX} 1$ and HX 3 can both reject heat to the environment. This is necessary in order to manage the thermal fronts as they emerge from the reservoirs. Ranges for all the design variables under consideration are given in Table 5. Two design scenarios are considered: an optimistic scenario, for which $\eta=0.99$, and a standard scenario with $\eta=0.90$ (i.e., achievable in principle with turbomachinery). Nominal values have been retained for the other loss factors.

\subsection{Pareto fronts and parallax plots}

The best designs emerging from the optimisation are shown in Fig. 6 in the form of 'Pareto fronts'. These are the leading edges of the design space in that all other solutions lie either below or to the left of these 
fronts. The Pareto fronts thus show the trade-off between the different objective functions and allow the designer to see the entire range of potential solutions.

As expected, there is a trade-off between efficiency and energy density (Fig. 6(a)), whereas Fig. 6(b) indicates that efficient designs are consistent with high power density. It is notable that roundtrip efficiency changes by only a small amount over a wide range of energy density for both the optimistic and standard scenarios. Thus, for example, a thermodynamic efficiency of $85 \%$ and energy density of $200 \mathrm{MJ} / \mathrm{m}^{3}$ could be achieved simultaneously in the optimistic case.

\subsection{Parallax plots}

Figure 7 is a so-called parallax plot, which compares values of the different design variables of the optimal solutions. To avoid overcrowding, only four designs have been plotted (Points 1 through 4 on Fig. 6), but by examining a parallax plot for the full Pareto front it is possible to draw out information about the best designs. For example, if all solutions converge on a narrow range of a particular parameter it suggests that the objective functions are sensitive to that parameter. (Note that the parameter ranges shown in the figure are taken from the full Pareto front.) From Fig. 7 it is apparent that:

(i) The main factor controlling the trade-off between efficiency and energy density is the utilisation, $\Pi$.

(ii) High polytropic efficiency, $(\eta=0.99)$, seems to correlate with low pressure ratio (typically around 7.5:1) and high $T_{1}$. A further parametric study, centred on the optimal designs, indicated that the optimiser had not quite converged: increasing the pressure ratio to $10: 1$ with a commensurate reduction in $T_{1}$ gave a very slight $(0.1 \%)$ improvement in efficiency. Nonetheless, the results shown in Fig. 7 suggest that almost the same performance can be achieved at lower cost - lower pressure ratio and higher $T_{1}$ imply cheaper components, notably CE, EC, HR and HX1.

(iii) Optimum discharge pressure ratios lie slightly below the charge pressure ratio.

(iii) Some parameters, notably the top temperature $T_{2}$ and hot reservoir discharged temperature, $T_{3}$, are 'saturating' at their limiting values, particularly for low $\eta$ cases. Although this suggests that the bounds on these parameters should be relaxed, this should only be done whilst also considering economic and other practical factors.

Some of these observations could have been deduced from the parametric studies or from simple thermodynamic considerations, but others (notably Point (ii)) are not so obvious and highlight the benefits of optimisation techniques.

\subsection{Comparison of loss distributions}

The optimal designs in the optimistic scenario have considerably higher efficiency than the nominal design (even when account is taken of the higher assumed value for $\eta$ ) and it is interesting to see how this has been achieved. Figure 8 compares the breakdown of losses for the nominal design (but with $\eta=0.99$ ) and for the design corresponding to Point 3 in Fig. 6. Most of the improvement evidently occurs by optimisation of the thermal store geometries and particle sizes. The associated reduction in heat exchanger loss is really due to the avoidance of exit losses that occur when the thermal fronts emerge from the stores (these losses are passed on to the heat exchangers, rather than being associated with the stores themselves). As expected, optimisation of the stores for high efficiency (i.e., Point 3) results in roughly a half-and-half split between thermal and pressure losses. The resulting geometry is perhaps a little unrealistic in that the short, fat reservoirs would be prone to uneven flow distributions through the packing, would require a larger footprint and would lead to 
manifold and pipework complications. Such difficulties need to be explored further, but it is possible that they can be mitigated by the use of segmented stores, as proposed by Crandall and Thatcher [20].

It is also apparent from Fig. 8 that a substantial part of the overall loss is due to heat leakage to and from the compressors and expanders. This merely reflects the assumed heat leakage factors of $2 \%$ for both $\mathrm{CE}$ and EC, and it is possible that lower heat leakage could be achieved in practice. Based on estimates from early prototypes and approximate (not fully non-dimensionalised) scaling, Howes [12] argues that heat leakage for a $2 \mathrm{MW}$ machine should be negligible, which according to Fig. 8 would reduce thermodynamic losses by another $3.5 \%$.

\section{Conclusions}

A study of thermodynamic aspects of pumped thermal electricity storage (PTES) has been presented, based on steady flow analysis of the compression and expansion devices coupled with a Schumann-style model of the hot and cold thermal stores. Parametric studies reveal that there are optimum values for some design variables, whilst others either lead to a trade-off between efficiency and energy density or can be varied so as to improve both these quantities together. Sensitivity of performance to the various loss factors has also been considered. Since the charge-discharge cycle for a PTES system involves two compressions and two expansions, the roundtrip efficiency is (not unexpectedly) sensitive to the loss factors for these processes. In this respect, a better understanding is required of the various sources of irreversibility within reciprocating devices.

Multi-objective optimisation has been applied to generate trade-off surfaces known as Pareto fronts. These show that curves of roundtrip efficiency vs. energy density are relatively flat over a considerable range, so that high energy density can be attained with only a modest efficiency penalty. In the optimised designs, losses associated with pressure drop and irreversible heat transfer in the stores are only a few percent, so the success of PTES is likely to hinge upon compressor and expander performance. Predicted efficiencies and storage densities obviously depend on the assumed loss factors; with an 'optimistic' set of parameters that might be achievable with reciprocating devices, the thermodynamic round-trip efficiency could exceed $85 \%$ whilst the system simultaneously achieves an energy density almost an order of magnitude greater than that for CAES. With reasonable estimates for mechanical and electrical losses, this would give an overall roundtrip efficiency (i.e., electricity out over electricity in) of just under $70 \%$. On the other hand, if the compressors and expanders could only achieve efficiencies typical of turbomachinery then the overall roundtrip efficiency is unlikely to exceed $50 \%$.

\section{Acknowledgement}

The work described in this paper was undertaken as part of a project funded by the UK Engineering and Physical Sciences Research Council (EPSRC Grant No. EP/J006246/1). The first author was supported by an EPSRC-funded studentship. All authors gratefully acknowledge this support. 


$\begin{array}{llll}A & \text { area, } \mathrm{m}^{2} & \alpha & \text { heat leakage factor, } \mathrm{d} q / \mathrm{d} w \\ c_{s} & \text { solid specific heat capacity, } \mathrm{J} \mathrm{kg}^{-1} \mathrm{~K}^{-1} & \alpha_{t} & \text { gas thermal diffusivity, } \mathrm{m}^{2} \mathrm{~s}^{-1} \\ c_{p} & \text { gas specific heat capacity, } \mathrm{J} \mathrm{kg}^{-1} \mathrm{~K}^{-1} & \beta & \text { pressure ratio } \\ C_{f} & \text { friction coefficient in packed bed } & \gamma & \text { ratio of specific heats } \\ D & \text { reservoir diameter, } \mathrm{m} & \chi & \text { roundtrip efficiency } \\ d_{p} & \text { particle diameter, } \mathrm{m} & \varepsilon & \text { void fraction or clearance ratio } \\ E & \text { stored energy, } \mathrm{J} & \eta, \eta_{v} & \text { polytropic, volumetric efficiency } \\ f_{p} & \text { fractional pressure loss } & \Lambda & \text { dimensionless reservoir length } \\ G & \text { mass flow per unit area, } \mathrm{kg} \mathrm{s}^{-1} \mathrm{~m}^{-2} & \Pi & \text { reservoir utilisation factor } \\ h & \text { local heat transfer coefficient } & \rho & \text { density, } \mathrm{kg} \mathrm{m}^{-3} \\ \ell & \text { length scale, } \mathrm{m} & \rho_{\mathrm{E}} & \text { energy density, } \mathrm{J} \mathrm{m}^{-3} \\ L & \text { reservoir length, } \mathrm{m} & \rho_{\mathrm{P}} & \text { power per unit flowrate, } \mathrm{J} \mathrm{m}^{-3} \\ M_{s} & \text { mass of storage material, } \mathrm{kg} & \sigma & \text { valve-to-piston open area ratio } \\ \dot{m} & \text { gas mass flow rate, } \mathrm{kg} \mathrm{s} \mathrm{s}^{-1} & \tau & \text { time scale, } \mathrm{s}, \text { or temperature ratio }\end{array}$

$p \quad$ pressure, $\mathrm{Pa}$

$q \quad$ heat transfer per unit mass

$r_{v} \quad$ volume (compression) ratio

$\dot{S}_{\text {irr }} \quad$ entropy generation rate, $\mathrm{J} \mathrm{K}^{-1} \mathrm{~s}^{-1} \quad \mathrm{c}, \mathrm{h} \quad$ cold, hot

St Stanton number chg charge

$S_{v} \quad$ surface area to volume ratio, $\mathrm{m}^{-1}$ dis discharge

$t$ time, $\mathrm{s}$ g gas

$T$ temperature, $\mathrm{K} \quad \mathrm{S} \quad$ solid

$V_{s} \quad$ swept volume, $\mathrm{m}^{3} \quad$ CE compressor-expander

w specific work, $\mathrm{J} \mathrm{kg}^{-1} \quad$ EC expander-compressor

$\dot{W} \quad$ power input/output, $\mathrm{W}$

$1-4$ points on cycle, as shown in Fig. 1

3 Other symbols are defined in the text where they are used.

\section{Appendix A: Estimation of Loss Parameters}

The intention of this section is to estimate minimum values of the various loss factors that might be achieved with custom-built reciprocating devices. These estimates are rather approximate and further work is clearly required, some of which is currently underway (see, for example, Ref. [21]).

\section{A1 Volumetric efficiency}

Figure 9 shows a $p-V$ diagram for an ideal reciprocating device. As shown, the device is operating as a compressor; the direction of the processes would be reversed for an expander. In the absence of heat loss and irreversibility, Processes A-B and C-D are isentropic. The volumetric compression ratio is thus,

$$
r_{v}=\frac{V_{A}}{V_{B}}=\frac{V_{D}}{V_{C}}=\beta^{1 / \gamma} .
$$

If inlet valve pressure losses are small (see Section A2 below) then the mass of gas entering the cylinder during the induction stroke (D-A) is,

$$
m=\rho_{i}\left(V_{A}-V_{D}\right)=\rho_{i} V_{A}\left(1-\varepsilon r_{v}\right),
$$


where $\rho_{i}$ is the inlet density and $\varepsilon$ is the clearance volume ratio, $\left(V_{C} / V_{A}\right)$. The volumetric efficiency is thus,

$$
\eta_{v}=\frac{m}{\rho_{i} V_{s}}=\frac{1-\varepsilon r_{v}}{1-\varepsilon},
$$

where $V_{s}=(1-\varepsilon) V_{A}$ is the swept volume. The same expression may be applied to expanders provided the density in Eq. (A3) is replaced with the exit value. Eq. (A3) effectively gives the pressure ratio / mass flow characteristic of the device. In real devices, Eq. (A3) would need to be modified to account for valve pressure losses, mixing of the induced and residual gas, and non-isentropic Processes A-B and C-D.

\section{A2 Valve pressure losses}

Valve losses will be minimised if the valves open and close rapidly and are actuated when there is zero pressure difference across them. Pressure traces for the prototype devices reported in Ref. [12] bear a remarkable similarity to the $p-V$ diagram of Fig. 9 and suggest that this ideal might well be achievable in practice. Under these circumstances, the instantaneous total pressure drop may be estimated as,

$$
\Delta p=\frac{1}{2} \rho v_{p}^{2}\left(\frac{1-\sigma}{\sigma}\right)^{2},
$$

where $v_{p}$ is the instantaneous piston speed and $\sigma$ is the ratio of the valve free-flow area (including the effects of venae contractae) to piston area. Strictly, $\Delta p$ should be flow-averaged by integrating over the valve open period but, in the spirit of engineering estimates, it is computed here on the basis of the piston maximum velocity. Normalising by the local pressure then gives,

$$
f_{p}=\frac{\Delta p}{p}=\frac{\omega^{2} s^{2}}{8 R T}\left(\frac{1-\sigma}{\sigma}\right)^{2} .
$$

The fractional pressure losses for the valves computed from Eq. (A5) for the nominal design are approximately $0.5 \%$ at exit from CE and inlet to EC (States 2 and 3 respectively in Fig. 1), 1\% at inlet to CE (State 1) and $1.4 \%$ at exit from EC (State 4).

\section{A3 Heat transfer losses in the compressors and expanders}

In theory, the time-mean heat leakage to or from the compressors and expanders can be reduced to very small levels with sufficient insulation and so heat leakage factors have been arbitrarily set to $2 \%$ for baseline calculations. However, even if the processes are globally adiabatic, an efficiency loss will still be incurred due to time-varying irreversible heat transfer to and from the cylinder walls. To estimate the magnitude of this loss, the irreversibility is divided into three parts as follows (estimates are made only for compression and it is assumed that similar loss factor apply for expanders).

(i) Heat transfer during induction and delivery. The gas entering the compressor is preheated by the cylinder walls prior to compression. Likewise, it is also cooled during the delivery stroke. The net effect is that heat is transferred from the high exit temperature down to the low inlet temperature, as illustrated schematically in Fig. 10. For simplicity it is assumed that the complete process ( 1 to 2 in the figure) is adiabatic and that the compression $\left(1^{\prime}\right.$ to $\left.2^{\prime}\right)$ is isentropic. Writing the wall temperature as $T_{w}=T_{1}+\theta\left(T_{2}-T_{1}\right)$, where $0<\theta<1$, the heat transferred to the gas during induction can be expressed as,

$$
Q=h S\left(T_{w}-T_{1}\right) \tau=h S \theta\left(T_{2}-T_{1}\right) \tau,
$$

where $h$ is some average heat transfer coefficient, $S$ is the average internal surface area and $\tau$ is the duration of the induction stroke. This heat transfer increases the enthalpy of the induced mass according to, 


$$
Q=m c_{p}\left(T_{1}^{\prime}-T_{1}\right)=\rho_{1} v_{p} A c_{p}\left(T_{1}^{\prime}-T_{1}\right) \tau,
$$

where $v_{p}$ is the average piston speed during induction and $A$ is the piston face area. Equating these two expressions for $Q$ gives,

$$
T_{1}^{\prime}-T_{1}=\operatorname{St} \theta \frac{S}{A}\left(T_{2}-T_{1}\right)=T_{2}^{\prime}-T_{2},
$$

where $S t$ is the Stanton number (based on the average piston speed) and the right hand equality stems from specifying that the overall process is adiabatic. By inspection of the $T-s$ diagram of Fig. 10, and making use of Eq. (A8), the isentropic efficiency is simply,

$$
\eta=\frac{T_{2}^{\prime} / T_{1}^{\prime}-1}{T_{2} / T_{1}-1}=1-f\left(\beta^{(\gamma-1) / \gamma}-1\right) \quad \text { where } \quad f=\operatorname{St} \theta \frac{S}{A} .
$$

Note that $f$ represents the heat transferred during induction as a fraction of the work transfer and this fraction is independent of the pressure ratio, as expected. The efficiency loss is larger at high pressure ratio, however, since the heat is then transferred across a higher temperature difference. The factor $f$ is difficult to determine with any confidence, but a crude estimate may be obtained by setting $\theta=1 / 2$ and approximating the Stanton number from turbulent pipe flow correlations. For the nominal design conditions given in Table 2, this gives $f=0.0025$, which translates to an efficiency loss of approximately $0.4 \%$ at a pressure ratio of $10: 1$.

(ii) Heat transfer during the compression and expansion strokes. Heat transfer also occurs during processes A-B and C-D of Fig. 9, but the use of a simple heat transfer coefficient is no longer appropriate in this case because of the interaction between heat and work transfers, and the inherently unsteady-state process that takes place during these processes. An excellent explanation of the underlying physics is given by Lawton [22], and a number of studies have attempted to quantify the effect in the context of gas springs. Early work by Lee [23] lead to the development of a simple analytical expression for the so-called hysteresis loss in gas springs. This has proved to fit experimental data to a reasonable extent, as reported by Kornhauser and Smith [24]. In recent work by Mathie et al. [21] an extended semi-analytical model was proposed for the full conjugate (solid-gas) thermal problem in gas-springs. The finite conductivity of the walls was shown to play a role, exacerbating losses in some cases. However, given the uncertainty associated with other loss parameters presented in this section, it suffices here to consider Lee's simpler approach. Lee's result is shown in Fig. 11, expressed as a decrement in polytropic efficiency by attributing half the hysteresis loss to the compression stroke, A-B. The horizontal axis in this figure is the Peclet number, $\mathrm{Pe}=\omega D_{h}^{2} / 2 \alpha_{t}$, where $D_{h}$ is the cylinder mean hydraulic diameter and $\alpha_{t}$ is the mean thermal diffusivity of the gas. Based on the dimensions and conditions listed in Tables 2 and 3, the Peclet number for the hot cylinder is 34000 , suggesting an efficiency loss of just $0.3 \%$. However, the thermal dissipation loss in a real compressor (as opposed to a gas spring) may be considerably larger because the eddying and turbulent motion generated by the inlet valve is likely to enhance the effective diffusivity. In this context, Lawton [22] found that good agreement between theory and experiment for heat transfer rates in a motored piston engine could be obtained by multiplying the gas conductivity by 25 . This would have the effect of reducing the Peclet number to 1360 and thereby increasing the loss to $1.6 \%$. It should be cautioned that other models (notably that presented in Ref. [21]) predict higher losses than this.

(iii) Mixing loss. Due to irreversibility and heat transfer during Process $\mathrm{A} \rightarrow \mathrm{D}$, the residual gas may end up at a different temperature to that entering the cylinder, thereby giving rise to an additional mixing loss. With the small losses suggested by (i) and (ii) above, this mixing loss can be shown to be extremely small (less than one tenth of a percent), but it may be significant if there were heat losses to the surroundings. 


\section{References}

[1] C. N. Markides, "The role of pumped and waste heat technologies in a high-efficiency sustainable energy future for the UK," Appl. Therm. Eng., vol. 53, no. 2, pp. 197-209, May 2013.

[2] D. J. C. MacKay, Sustainable energy--without the hot air. Cambridge, England: UIT, 2009.

[3] H. Chen, Y. Ding, T. Peters, and F. Berger, "Energy storage and generation," US20090282840 A119Nov-2009.

[4] B. Wolf, "A method for storing and recovering energy," EP 1987299 B1.

[5] J. Ruer, "Installation and Methods for Storing and Recovering Electric Energy," WO/2008/14896212Dec-2008.

[6] J. Macnaghten and J. S. Howes, "Energy storage,” WO2009044139 A209-Apr-2009.

[7] C. Ohler, M. Mercangoez, and J. Hemrle, "Thermoelectric energy storage system and method for storing thermoelectric energy," EP2157317 B119-Jun-2013.

[8] B. Weissenbach, “Thermal Energy Storage Device,” EP0003980 A119-Sep-1979.

[9] A. White, G. Parks, and C. N. Markides, "Thermodynamic analysis of pumped thermal electricity storage," Appl. Therm. Eng., vol. 53, no. 2, pp. 291-298, May 2013.

[10] A. Thess, "Thermodynamic Efficiency of Pumped Heat Electricity Storage," Phys. Rev. Lett., vol. 111, no. 11, 2013.

[11] T. Desrues, J. Ruer, P. Marty, and J. F. Fourmigué, “A thermal energy storage process for large scale electric applications," Appl. Therm. Eng., vol. 30, no. 5, pp. 425-432, Apr. 2010.

[12] J. Howes, "Concept and Development of a Pumped Heat Electricity Storage Device," Proc. IEEE, vol. 100, no. 2, pp. 493-503, Feb. 2012.

[13] A. J. White, J. D. McTigue, and C. N. Markides, "Wave propagation and thermodynamic losses in packed--bed thermal reservoirs for energy storage," Appl. Energy, In Press.

[14] A. J. White, "Loss analysis of thermal reservoirs for electrical energy storage schemes," Appl. Energy, vol. 88, no. 11, pp. 4150-4159, 2011.

[15] R. Kurz and K. Brun, "Efficiency Definition and Load Management for Reciprocating and Centrifugal Compressors," pp. 1435-1445, Jan. 2007.

[16] A. J. White, "Thermodynamic analysis of the reverse Joule-Brayton cycle heat pump for domestic heating," Appl. Energy, vol. 86, no. 11, pp. 2443-2450, 2009.

[17] J. A. Willmott, Dynamics of Regenerative Heat Transfer. CRC Press, 2001.

[18] R. Holdich, Fundamentals of Particle Technology. Midland Information Technology and Publishing, 2002.

[19] K. Deb, A. Pratap, S. Agarwal, and T. Meyarivan, "A fast and elitist multiobjective genetic algorithm: NSGA-II," IEEE Trans. Evol. Comput., vol. 6, no. 2, pp. 182-197, Apr. 2002.

[20] D. M. Crandall and E. F. Thacher, "Segmented thermal storage," Sol. Energy, vol. 77, no. 4, pp. 435440, Oct. 2004.

[21] R. Mathie, C. N. Markides, and A. White, "A Framework for the Analysis of Thermal Losses in Reciprocating Compressors and Expanders," in Heat Transfer Engineering, Malta, 2012, pp. 337354.

[22] B. Lawton, "Effect of Compression and Expansion on Instantaneous Heat Transfer in Reciprocating Internal Combustion Engines," Proc. Inst. Mech. Eng. Part J. Power Energy, vol. 201, no. 3, pp. 175186, 1987.

[23] K. Lee, "A Simplistic Model of Cyclic Heat Transfer Phenomena in Closed Spaces," Proc Intersoc Energy Convers Eng Conf U. S., vol. 2, Aug. 1983.

[24] A. A. Kornhauser and J. L. Smith, "The Effects of Heat Transfer on Gas Spring Performance," J. Energy Resour. Technol., vol. 115, no. 1, p. 70, 1993. 
Figure 1: Layout of PTES system and corresponding $T-s$ diagram. Key: BV buffer vessel; HR/CR hot/cold reservoir; $\mathrm{CE} / \mathrm{EC}$ reversible compressor-expanders; $\mathrm{HX} 1 / \mathrm{HX} 3$ heat exchangers. The $T-s$ diagram shows the discharge cycle operating at a lower pressure ratio, with heat rejection from both HX1 and HX3.

Figure 2: Typical temperature profiles in a cold reservoir for cyclic operation with a utilisation factor of $\Pi=0.75$. The profiles are shown at various stages through the cycle. Differences between the solid and chain lines highlight the impact of the temperature-dependent solid heat capacity.

Figure 3: Sensitivity of thermodynamic round-trip efficiency to compressor and expander loss parameters.

Figure 4: Variation of roundtrip efficiency and energy density with operational parameters. Left: variation with $T_{1}, T_{3}$ and charging pressure ratio, $\beta_{\text {chg }}$ ( $T_{1}$ and $T_{3}$ fixed). Right: variation with $\beta_{\text {chg }}\left(T_{2}\right.$ and $T_{4}$ fixed), discharge pressure ratio, $\beta_{\mathrm{dis}}$, and utilisation factor $\Pi$. For each curve the open and solid square symbols indicate the minimum and maximum values of each parameter in accord with Table 4 .

Figure 5: Variation of thermodynamic roundtrip efficiency with geometric parameters. Minimum, nominal and maximum values of the parameters are given in Table 4 .

Figure 6: Pareto fronts (trade-off surfaces) emerging from the optimisation.

Figure 7: 'Parallax' plots showing the combinations of design parameters for the 'best' designs, as defined by data Points 1 to 4 in Fig. 6.

Figure 8: Comparison of loss distributions for the nominal design (but with $\eta=0.99$ ) and an optimised design (Point 3 in Fig. 6). Thermal losses refer to heat transfer irreversibility; heat leakage losses are due to heat exchange with the environment above or below ambient temperature.

Figure 9: $p-V$ diagram for an ideal reciprocating device. As shown the device is operating as a compressor; the cycle would be reversed for an expander.

Figure 10: simplified model of the irreversibility associated with preheating during induction and cooling during delivery, shown for a compression process. The overall Process 1-2 is assumed adiabatic.

Figure 11: Estimate of the hysteresis loss during the compression and expansion processes of Fig. 9, based on the model of Lee [23].

\section{TABLE CAPTIONS}

Table 1: Comparison of maximum exergetic flows per unit mass and per unit volume of working fluid for various technologies. (The dead state is taken as $20^{\circ} \mathrm{C}$ and 1 bar.)

Table 2: Hot and cold reservoir details for a nominal 16 MWh PTES system. The storage material is $\mathrm{Fe}_{3} \mathrm{O}_{4}$ (density 5.175 tonne $/ \mathrm{m}^{3}$ ) in the form of a packed bed with an assumed void fraction of 0.35

Table 3: Details of the compression-expansion devices for a nominal power of $2 \mathrm{MW}$. A clearance ratio $\left(V_{\min } / V_{\max }\right)$ of 0.05 and a cylinder aspect ratio (stroke/diameter) of 0.25 and have been assumed for both devices.

Table 4: Nominal, minimum and maximum values of quantities varied for the parametric study. Note that the loss factors are for each compressor/expander, $\mathrm{CE}$ and $\mathrm{EC}$, with half the pressure loss assigned to each side of the device.

Table 5: Lower and upper bounds for parameters used in optimisation. Ambient temperature is $T_{0}=298 \mathrm{~K}$. 
2 Table 1: Comparison of maximum exergetic flows per unit mass and per unit volume of working fluid for various 3 technologies. (The dead state is taken as $20^{\circ} \mathrm{C}$ and 1 bar.)

\begin{tabular}{|l||c|c|c|c|}
\hline & CAES & PHS & PTES (Joule) & Rankine Cycle \\
\hline \hline Working fluid & air & water & argon & steam \\
\hline Operating conditions & $20{ }^{\circ} \mathrm{C}, 100 \mathrm{bar}$ & $500 \mathrm{~m}$ & $500{ }^{\circ} \mathrm{C}, 10.5 \mathrm{bar}$ & $500{ }^{\circ} \mathrm{C}, 100 \mathrm{bar}$ \\
\hline Exergy per unit mass & $390 \mathrm{~kJ} / \mathrm{kg}$ & $4.9 \mathrm{~kJ} / \mathrm{kg}$ & $250 \mathrm{~kJ} / \mathrm{kg}$ & $1400 \mathrm{~kJ} / \mathrm{kg}$ \\
\hline Exergy per unit volume & $46 \mathrm{MJ} / \mathrm{m}^{3}$ & $4.9 \mathrm{MJ} / \mathrm{m}^{3}$ & $1.6 \mathrm{MJ} / \mathrm{m}^{3}$ & $43 \mathrm{MJ} / \mathrm{m}^{3}$ \\
\hline
\end{tabular}

Table 2: Hot and cold reservoir details for a nominal $16 \mathrm{MWh}$ PTES system. The storage material is $\mathrm{Fe}_{3} \mathrm{O}_{4}\left(\mathrm{den}_{\mathrm{ity}}\right.$ 5.175 tonne $/ \mathrm{m}^{3}$ ) in the form of a packed bed with an assumed void fraction of 0.35 .

\begin{tabular}{|c|c|c|c|c|c|c|c|c|}
\hline & & $\begin{array}{c}P \\
\text { (bar) }\end{array}$ & $\begin{array}{l}T \\
(\mathrm{~K})\end{array}$ & $\begin{array}{c}\rho_{g} \\
\left(\mathrm{~kg} \mathrm{~m}^{-3}\right)\end{array}$ & $\begin{array}{r}M_{g} \\
(\mathrm{~kg})\end{array}$ & $\begin{array}{c}\bar{c}_{s} \\
\left(\mathrm{~J} \mathrm{~kg}^{-1} \mathrm{~K}^{-1}\right)\end{array}$ & $\begin{array}{c}M_{s} \\
\text { (tonne) }\end{array}$ & $\begin{array}{c}V \\
\left(\mathrm{~m}^{3}\right)\end{array}$ \\
\hline \multirow[t]{2}{*}{ HOT } & Chg & $10.5 \mathrm{bar}$ & 778 & 6.5 & 162 & \multirow[t]{2}{*}{860} & \multirow[t]{2}{*}{238} & \multirow[t]{2}{*}{71} \\
\hline & Dis. & $10.5 \mathrm{bar}$ & 310 & 16.3 & 405 & & & \\
\hline \multirow[t]{2}{*}{ COLD } & Chg. & $1.05 \mathrm{bar}$ & 123 & 4.1 & 168 & \multirow[t]{2}{*}{520} & \multirow[t]{2}{*}{394} & \multirow[t]{2}{*}{117} \\
\hline & Dis. & $1.05 \mathrm{bar}$ & 310 & 1.63 & 67 & & & \\
\hline
\end{tabular}

Table 3: Details of the compression-expansion devices for a nominal power of $2 \mathrm{MW}$. A clearance ratio $\left(V_{\min } / V_{\max }\right)$ of 0.05 and a cylinder aspect ratio (stroke/diameter) of 0.25 and have been assumed for both devices.

\begin{tabular}{|l|c|c|c|c|c|c|}
\hline & $\begin{array}{c}\text { Speed } \\
(\mathrm{RPM})\end{array}$ & $\begin{array}{c}\boldsymbol{V}_{\mathbf{s}}(\mathbf{t o t a l}) \\
\left(\mathrm{m}^{3}\right)\end{array}$ & $\boldsymbol{N}_{\text {cyl }}$ & $\begin{array}{c}\boldsymbol{D} \\
(\mathrm{m})\end{array}$ & $\begin{array}{c}\text { Stroke } \\
(\mathrm{m})\end{array}$ & $\begin{array}{c}\text { Clearance } \\
(\mathrm{mm})\end{array}$ \\
\hline \hline Hot cylinders (CE) & 1200 & 0.50 & 6 & 0.75 & 0.19 & 10 \\
\hline Cold cylinders (EC) & 1200 & 0.20 & 6 & 0.55 & 0.14 & 7.4 \\
\hline
\end{tabular}

Table 4: Nominal, minimum and maximum values of quantities varied for the parametric study. Note that the loss factors are for each compressor/expander, CE and EC, with half the pressure loss assigned to each side of the device.

\begin{tabular}{|l||c|c|c||c|c|c|c|c||c|c|c|c|}
\hline \multicolumn{1}{|c||}{} & \multicolumn{3}{c||}{ Loss Factors } & \multicolumn{4}{c||}{ Operating Conditions } & \multicolumn{3}{c|}{ Geometric Parameters } \\
\hline & $f_{\mathrm{p}}$ & $1-\eta$ & $\alpha$ & $\begin{array}{c}T_{1} \\
(\mathrm{~K})\end{array}$ & $\begin{array}{c}T_{3} \\
(\mathrm{~K})\end{array}$ & $\beta_{\mathrm{chg}}$ & $\beta_{\mathrm{dis}}$ & $\Pi$ & $(L / D)_{\mathrm{CR}}$ & $(L / D)_{\mathrm{HR}}$ & $\begin{array}{c}d_{p}^{\mathrm{CR}} \\
(\mathrm{cm})\end{array}$ & $\begin{array}{c}d_{p}^{\mathrm{HR}} \\
(\mathrm{cm})\end{array}$ \\
\hline Nominal & 0.02 & 0.02 & 0.02 & 310 & 310 & 10.0 & 10.0 & 0.75 & 1.00 & 1.00 & 2.0 & 2.0 \\
\hline Min & 0.00 & 0.00 & 0.00 & 273 & 273 & 5.00 & 5.00 & 0.50 & 0.10 & 0.10 & 0.5 & 0.5 \\
\hline Max & 0.04 & 0.04 & 0.04 & 347 & 347 & 15.0 & 15.0 & 1.00 & 1.90 & 10.0 & 3.5 & 3.5 \\
\hline
\end{tabular}

Table 5: Lower and upper bounds for parameters used in optimisation. Ambient temperature is $T_{0}=298 \mathrm{~K}$.

\begin{tabular}{|l|c|c|c|c|c|c|c|c|}
\hline & $L / D$ & $d_{p}(\mathrm{~cm})$ & $\Pi$ & $\beta$ & $T_{1}(\mathrm{~K})$ & $T_{2}(\mathrm{~K})$ & $T_{3}(\mathrm{~K})$ & $T_{4}(\mathrm{~K})$ \\
\hline \hline Lower bound & 0.10 & 0.50 & 0.10 & 2.00 & $T_{0}+10$ & $T_{3}+50$ & $T_{0}+10$ & 103 \\
\hline Upper bound & 10.0 & 10.0 & 0.99 & 20.0 & 773 & 873 & 773 & $T_{1}-50$ \\
\hline
\end{tabular}


2
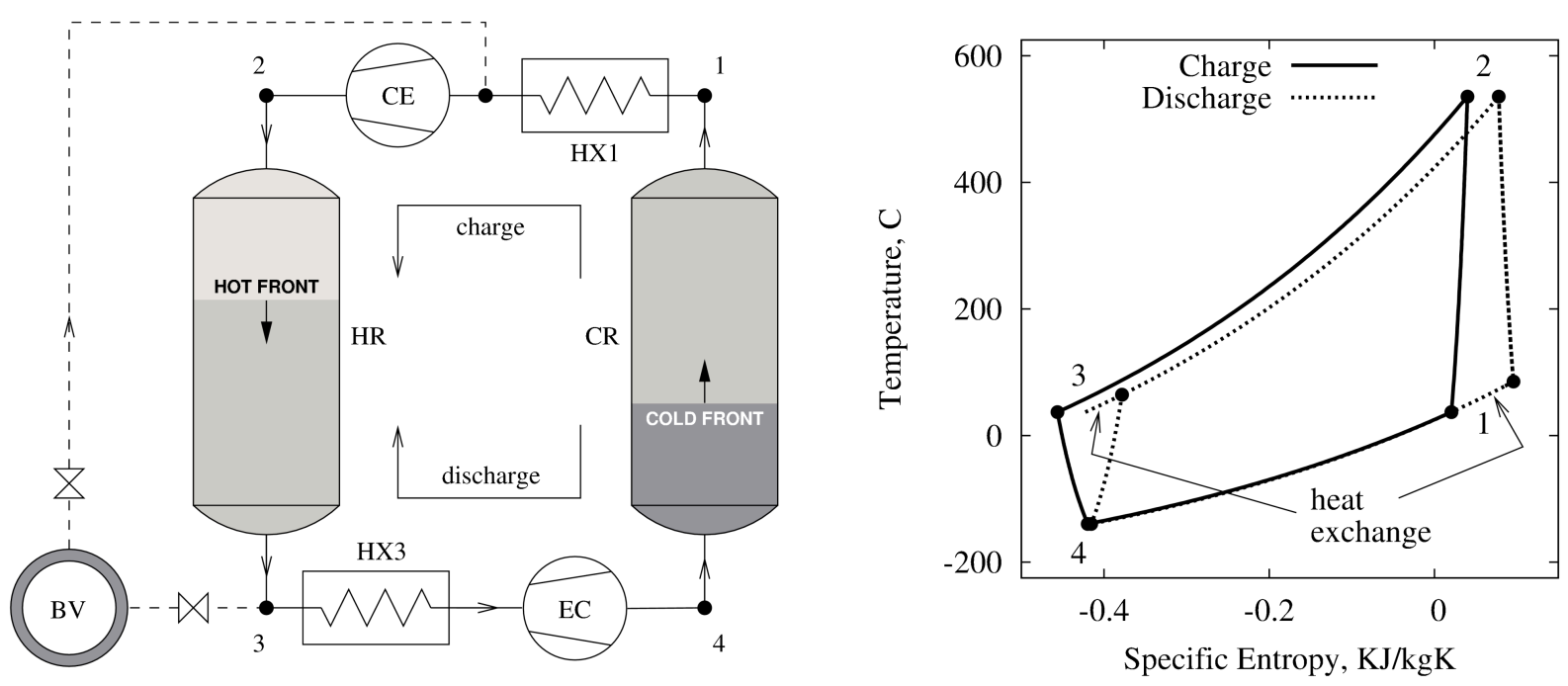

Figure 1: Layout of PTES system and corresponding $T-s$ diagram. Key: BV buffer vessel; HR/CR hot/cold reservoir; CE/EC reversible compressor-expanders; HX1/HX3 heat exchangers. The $T-s$ diagram shows the discharge cycle operating at a lower pressure ratio, with heat rejection from both HX1 and HX3.

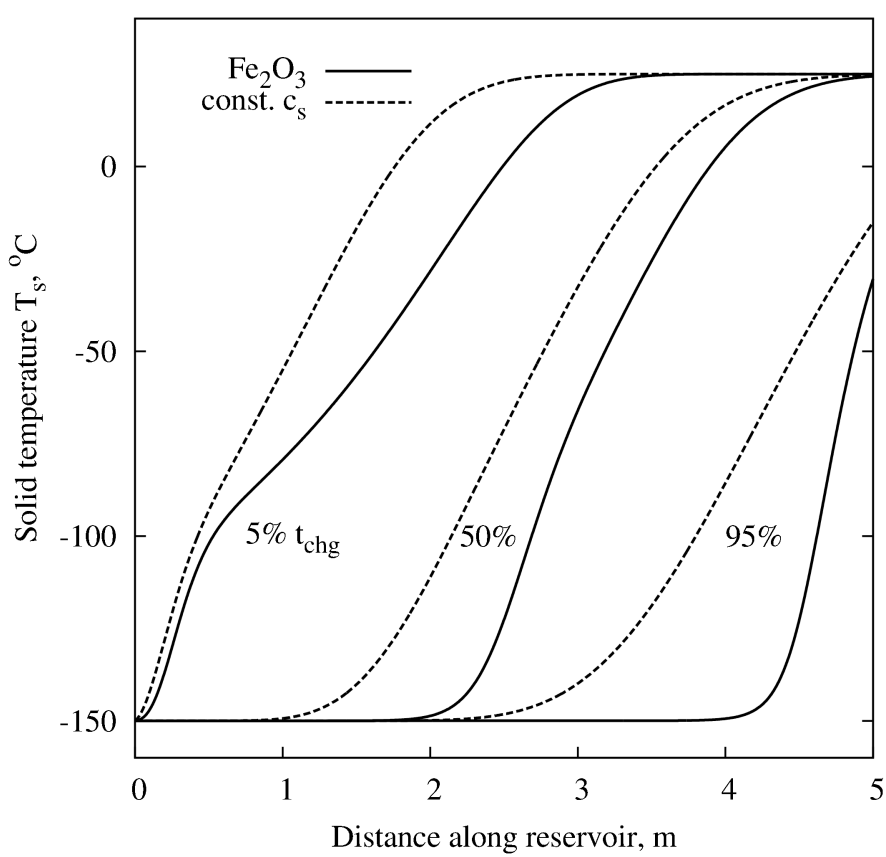

Figure 2: Typical temperature profiles in a cold reservoir for cyclic operation with a utilisation factor of $\Pi=0.75$. The profiles are shown at various stages through the cycle. Differences between the solid and chain lines highlight the impact of the temperature-dependent solid heat capacity. 


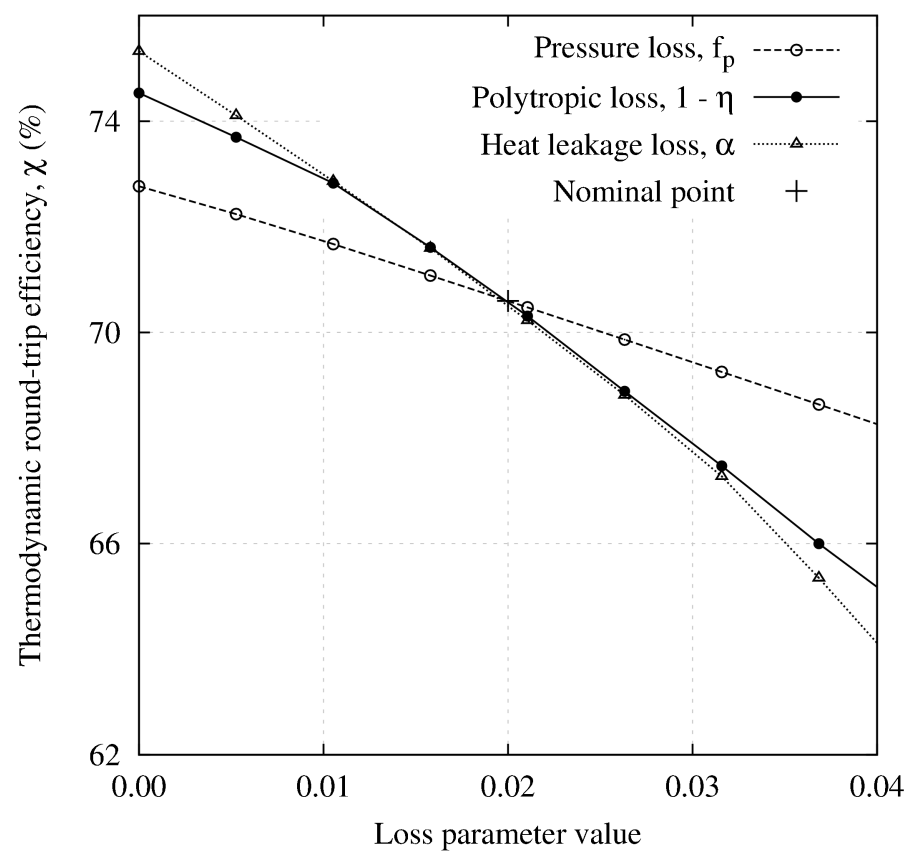

Figure 3: Sensitivity of thermodynamic round-trip efficiency to compressor and expander loss parameters.
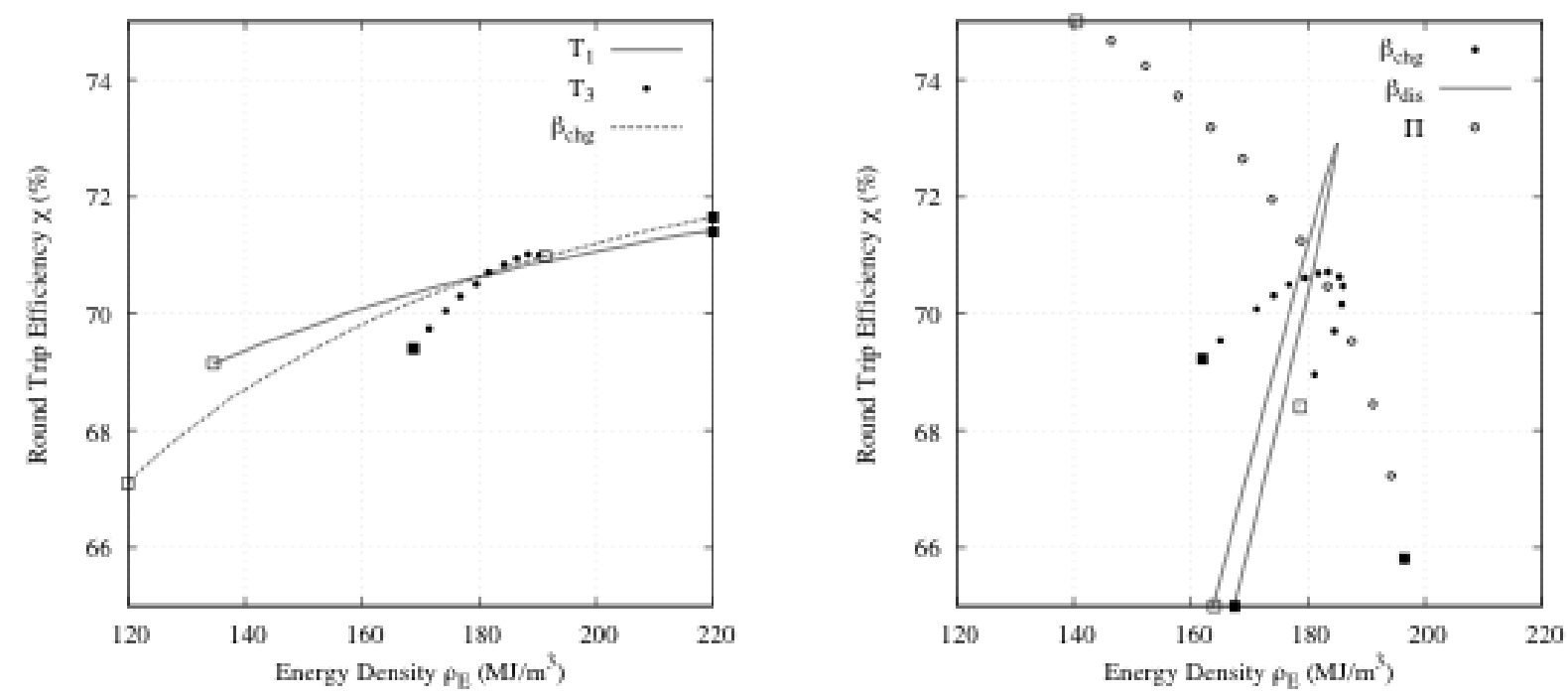

Figure 4: Variation of roundtrip efficiency and energy density with operational parameters. Left: variation with $T_{1}, T_{3}$ and charging pressure ratio, $\beta_{\mathrm{chg}}$ ( $T_{1}$ and $T_{3}$ fixed). Right: variation with $\beta_{\mathrm{chg}}\left(T_{2}\right.$ and $T_{4}$ fixed), discharge pressure ratio, $\beta_{\mathrm{dis}}$, and utilisation factor $\Pi$. For each curve the open and solid square symbols indicate the minimum and maximum values of each parameter in accord with Table 4 . 


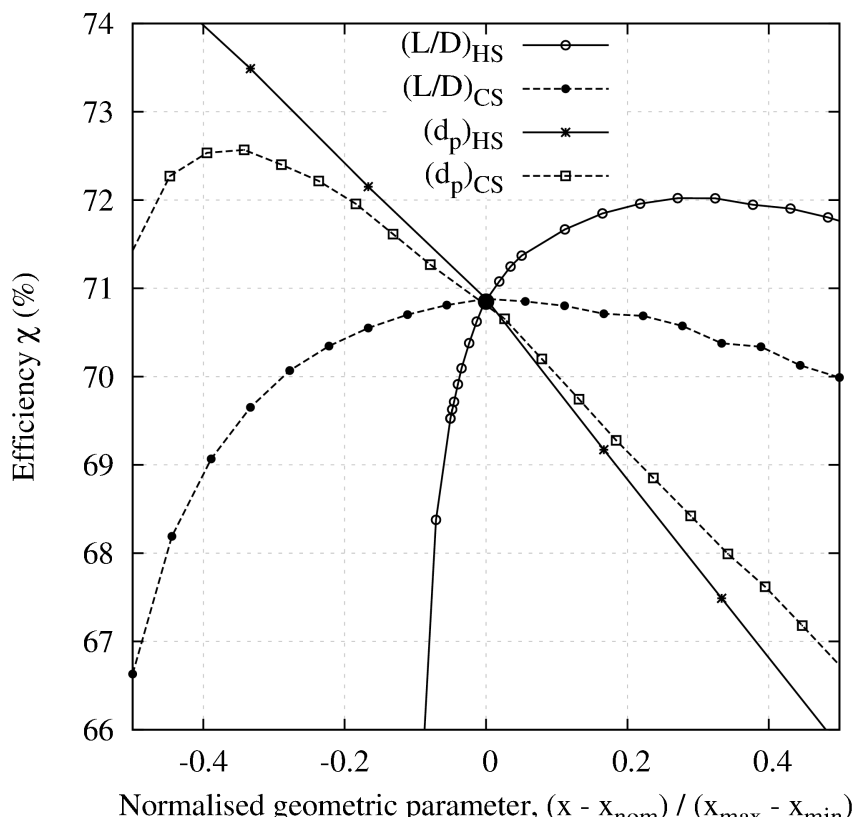

Figure 5: Variation of thermodynamic roundtrip efficiency with geometric parameters. Minimum, nominal and maximum values of the parameters are given in Table 4.

5

6

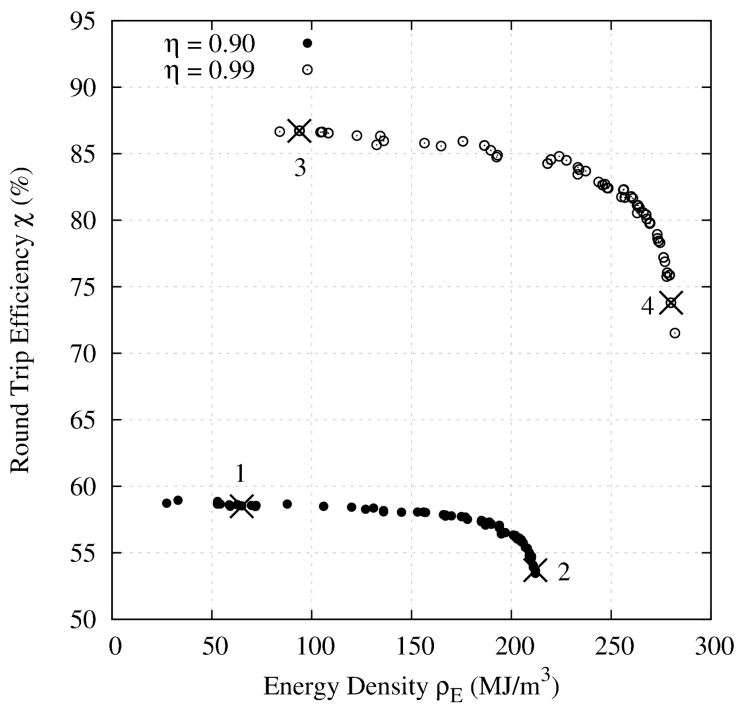

(a) Efficiency vs. Energy Density

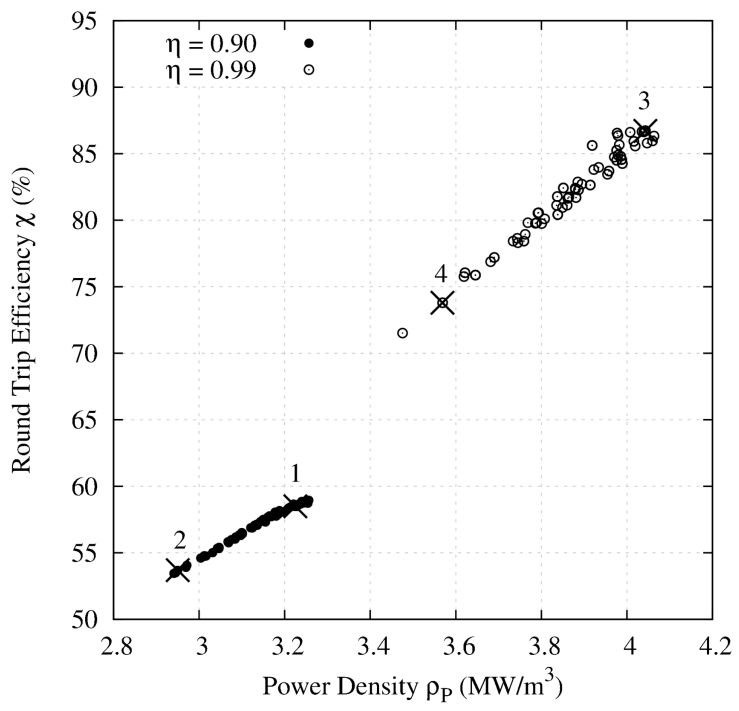

(b) Efficiency vs. Power Density

Figure 6: Pareto fronts (trade-off surfaces) emerging from the optimisation. 


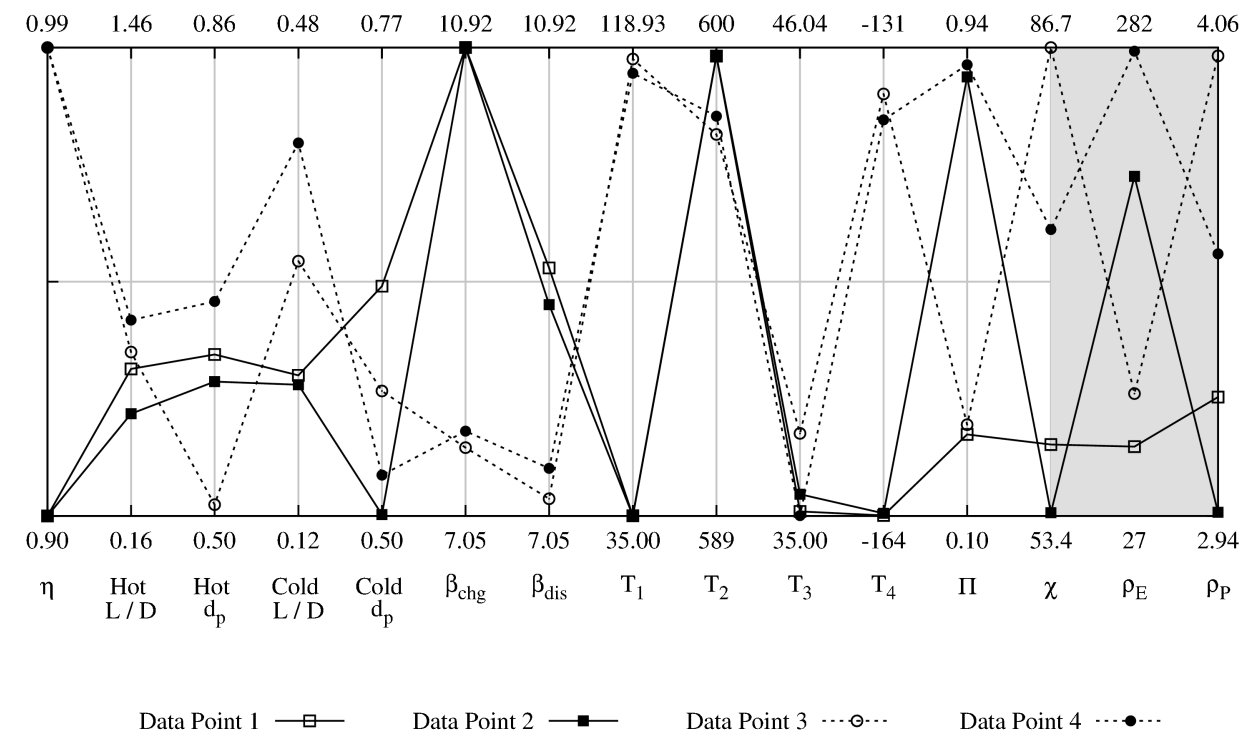

Figure 7: 'Parallax' plots showing the combinations of design parameters for the 'best' designs, as defined by data Points 1 to 4 in Fig. 6.

5

6

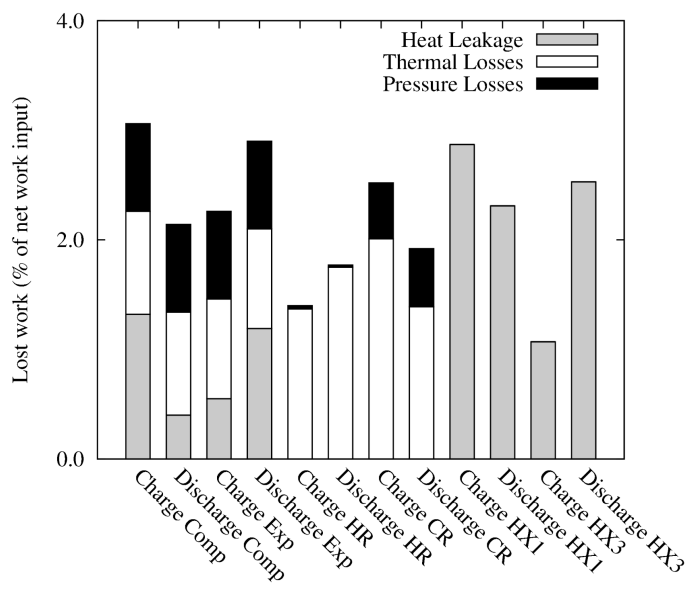

(a) Nominal Design

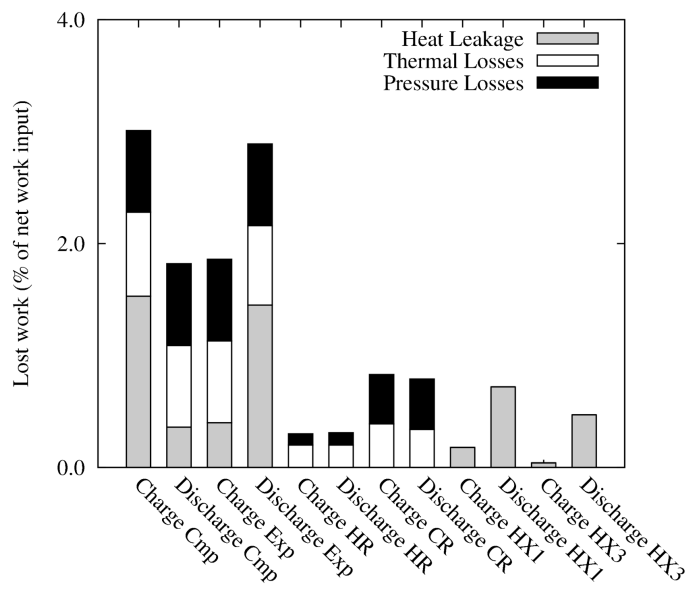

(b) Optimal Design (point 3)

Figure 8: Comparison of loss distributions for the nominal design (but with $\eta=0.99$ ) and an optimised design (Point 3 in Fig. 6). Thermal losses refer to heat transfer irreversibility; heat leakage losses are due to heat exchange with the environment above or below ambient temperature. 


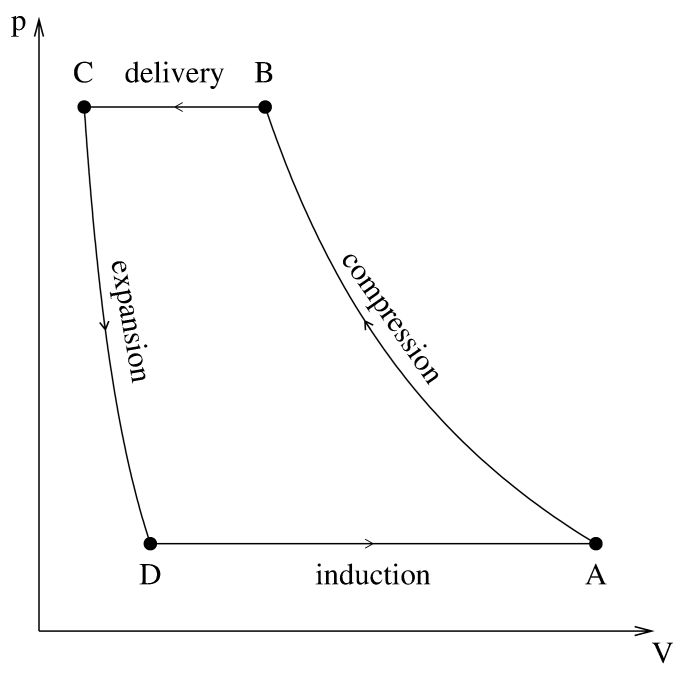

Figure 9: $p$ - $V$ diagram for an ideal reciprocating device. As shown the device is operating as a compressor; the cycle would be reversed for an expander.

5

6
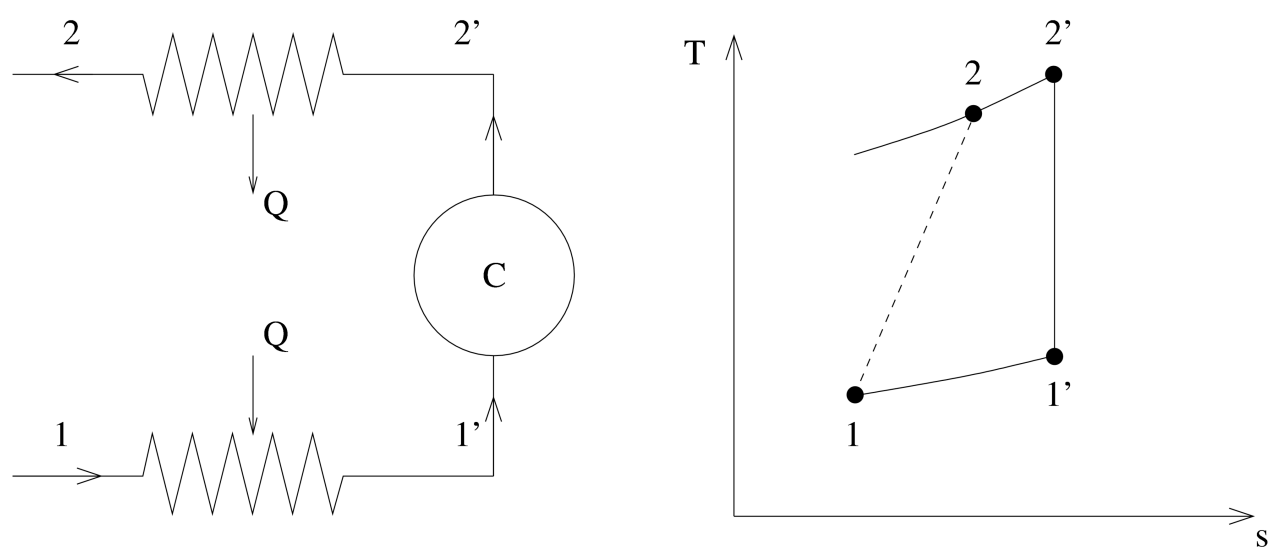

Figure 10: Simplified model of the irreversibility associated with preheating during induction and cooling during delivery, shown for a compression process. The overall Process 1-2 is assumed adiabatic.

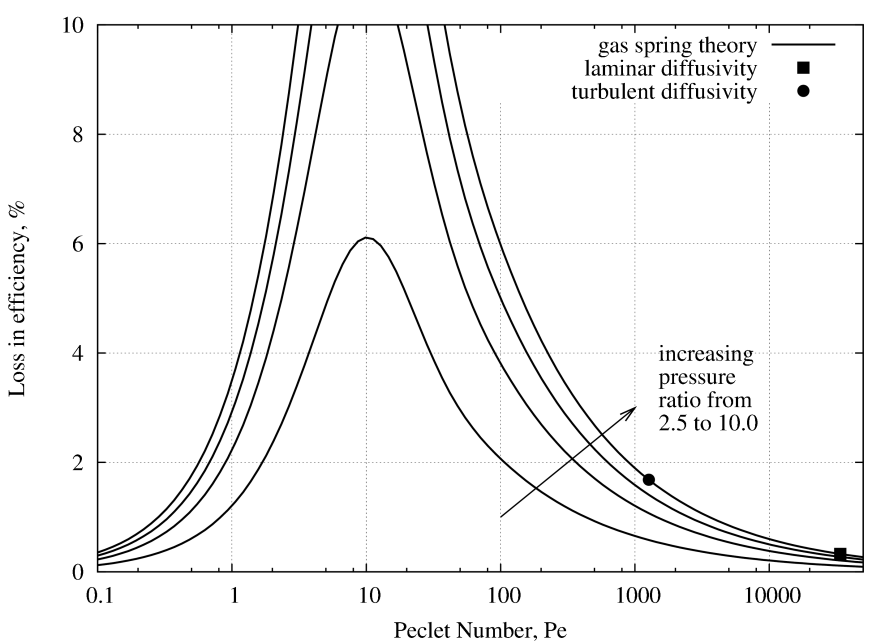

Figure 11: Estimate of the hysteresis loss during the compression and expansion processes of Fig. 9, based on the model of Lee [23]. 